\title{
Development of culturally appropriate pictorial cards to facilitate maternal health histories in rural Ghana
}

\author{
Amir Sabet Sarvestani ${ }^{\text {a }}$, Thomas O. Konney ${ }^{\mathrm{b}}$, Kathleen H. Sienko ${ }^{\mathrm{c}, \mathrm{d}, *}$ \\ a Design Science Program, University of Michigan, Ann Arbor, USA \\ ${ }^{\mathrm{b}}$ Department of Obstetrics and Gynecology, Komfo Anokye Teaching Hospital, Kumasi, Ghana \\ c Department of Mechanical Engineering, University of Michigan, Ann Arbor, USA \\ d Department of Biomedical Engineering, University of Michigan, Ann Arbor, USA
}

\section{A R T I C L E I N F O}

\section{Article history:}

Received 29 March 2013

Received in revised form 11 June 2013

Accepted 27 August 2013

\section{Keywords:}

Community health workers

Health record

Low-resource countries

Maternal health

Pictorial cards

Pregnancy complications

Referral decision making

Overall, 99\% of annual global maternal deaths occur in lowresource countries. Delays in identifying danger signs during pregnancy and in deciding appropriate actions contribute to this mortality trend [1]. Limited health education among community health workers (CHWs) and the high illiteracy rates prevalent in rural settings make recognition of pregnancy complications and subsequent referral decisions challenging. Home-based maternal health records and pictorial cards illustrating pregnancy complications have decreased the time associated with identifying at-risk cases, increased the diagnosis and referral of at-risk pregnant women [2], promoted self-care, and educated women in low-resource settings [2-4]. However, pregnant women and providers have encountered problems interpreting and relating to the illustrations [4].

The aim of the present study was to develop and evaluate culturally appropriate and easy-to-understand pictorial cards of common pregnancy complications. Institutional review boards at the University of Michigan and Ghana Health Service approved the study, and participants provided informed consent.

The original set of pictorial cards illustrating 16 pregnancy complications (Fig. 1) was evaluated using a 10-point Likert scale during interviews with pregnant women $(n=18)$ in the Sene and Kumasi

\footnotetext{
* Corresponding author at: University of Michigan, 2350 Hayward Street, 3116 G.G. Brown, Ann Arbor, MI 48109, USA. Tel.: + 1734647 8249; fax: +1 7346479379. E-mail address: sienko@umich.edu (K.H. Sienko).
}

Metropolitan Districts of Ghana. Participants scored their agreement with each illustration's ability to convey the intended complication, and commented on the cultural appropriateness. Based on feedback from participants and input from clinicians, midwives/nurses, and CHWs, 13 of the illustrations were revised and then all of the illustrations were re-evaluated within the same communities $(n=45)$. The scores were analyzed using SPSS version 20 (IBM, Armonk, NY, USA) and tested for significance $(P<0.05)$, applying independent-samples $t$ test.

Ten of the revised illustrations were found to be significantly improved. The revisions ranged from minimal to extensive; the most commonly requested change was a facial expression that communicated pain. An example of cultural appropriateness is that participants actually associated fever with a thermometer placed in the armpit, whereas the original illustration of fever showed a thermometer placed under the tongue. Two revised images (fainting and difficulty breathing), which were difficult to illustrate because of the physical movements associated with the complications, showed lower scores but the decrease was statistically insignificant.

The study largely relied on input from a small sample of pregnant women from 2 rural regions in Ghana. The inclusion of additional endusers and ethnic groups would probably improve the acceptance of pregnancy complication representations.

Pictorial cards could potentially be used by community health workers to elicit information during a health history interview, facilitate recognition of pregnancy complications, and improve referral communications between primary and referral healthcare centers [4]. The present study reinforces the importance of involving end-users throughout the development of a diagnostic aid that has major cultural implications.

\section{Conflict of interest}

The authors have no conflicts of interest.

\section{References}

[1] Thaddeus S, Maine D. Too far to walk: maternal mortality in context. Soc Sci Med 1994;38(8):1091-110.

[2] Shah PM, Selwyn BJ, Shah K, Kumar V. Evaluation of the home-based maternal record: a WHO collaborative study. Bull World Health Organ 1993;71(5):535-48.

[3] Turner KE, Fuller S. Patient-Held Maternal and/or Child Health Records: Meeting the Information Needs of Patients and Healthcare Providers in Developing Countries? Online J Public Health Inform 2011;3(2).

[4] Kumar V, Datta N. Home-based mothers' health records. World Health Forum 1988;9(1):107-10. 


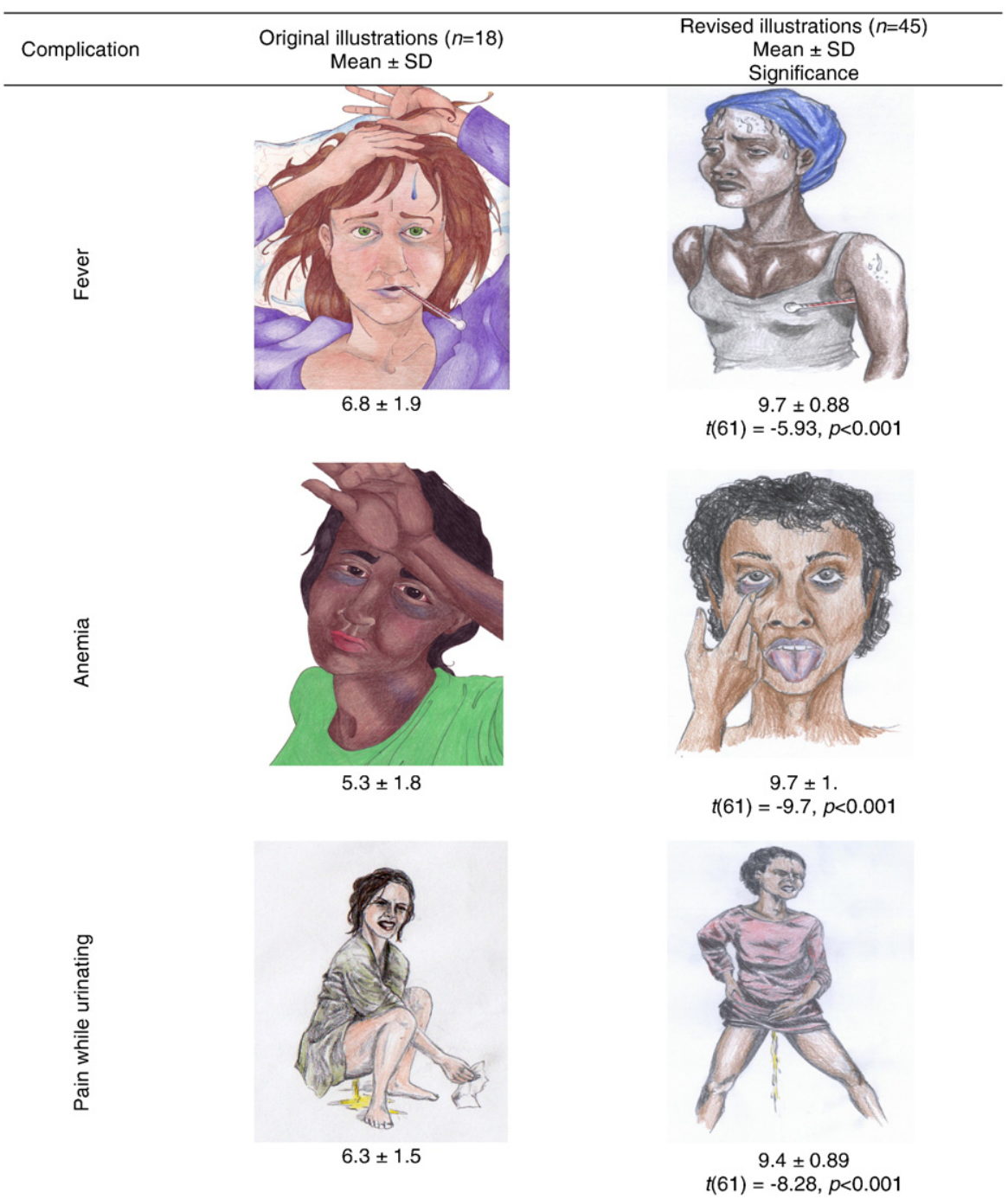

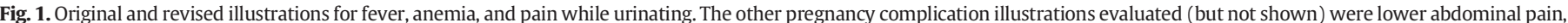

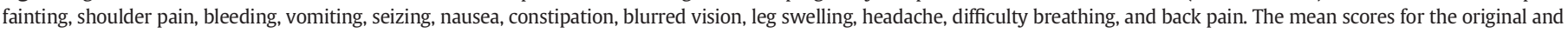

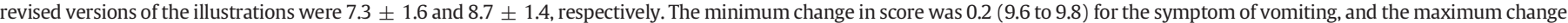

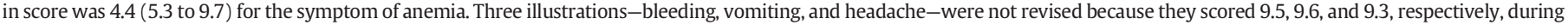
the first evaluation. 